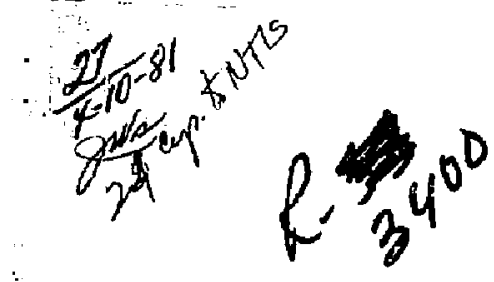

UCID. 18988

\title{
MASTR
}

A TWO-WAVE LENGTH HENe LASER INTERFEROMETER

E. H. A. Granneman

March 24, 1981

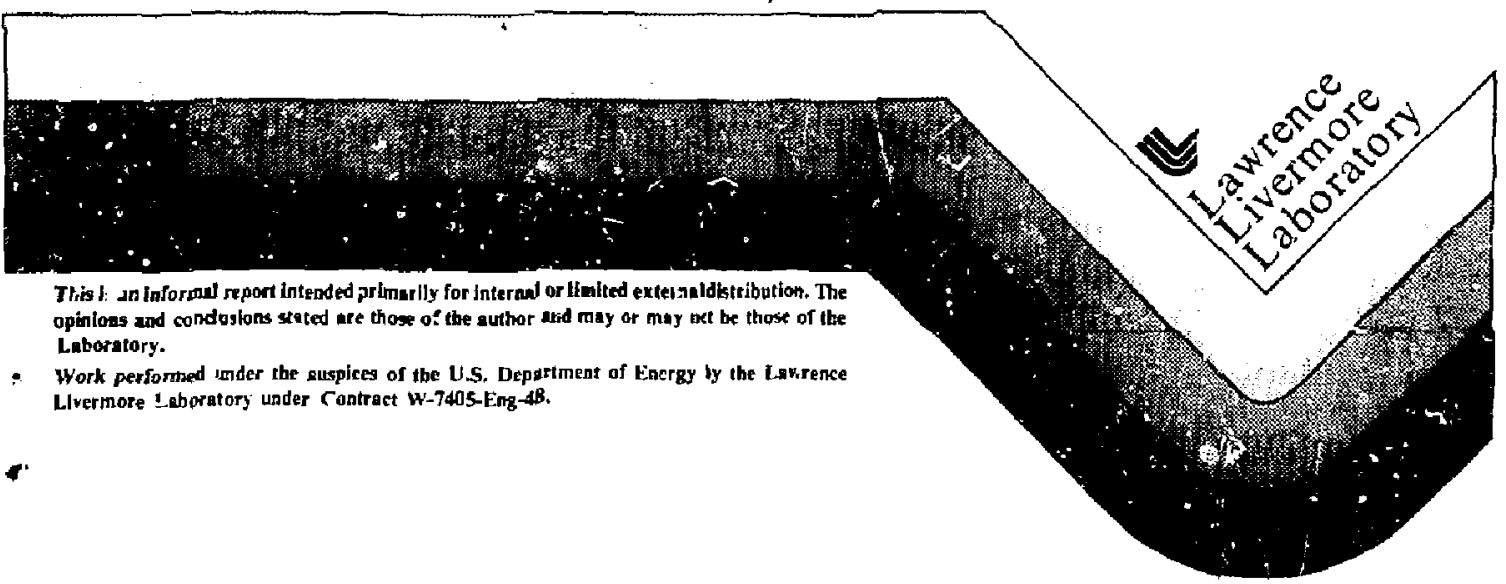

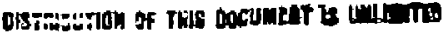




\section{A TWO-WAVELENGTH HENE LASER INTERFEROMETER}

E. B. A. Granneman*

ABSTRACT

This paper presents an interferometer set-up in which two wavelengths are used simultaneously. This enables one to determine separately the piase shifts caused by changes in plasma density and by mechanical vibrations of the interferometer structure.

1. The BETA II Inter ferometer:

At present the plasma density in the BETA II field reserved plasma ring is measured by means of a HeNe laser interferometer operating at a wavelength of $0.6328 \mu \mathrm{m}$. This system consists of the usual combination of a rest leg going through the plasma and a reference lez outside the plasma (see figure 1). The system is double pass, which tuhances the sensitivity by a factor of two. By giving the reference and cest legs the same length, the spot size of both beams at the point of recombination is the same. The horizontal and vertical dimensions are roughly 3.0 and $2.5 \mathrm{~m}$, respectively. The laser and all the optical components are mounted inside a rigid, closed structure made of an epoxy fiberglass laminated sheet (G-10). This structure is connected to tipe machine through a number of vibrationaily isolated mounts placed underneath the horizontal bar of the system.

The fringe pattern registered by the detectors is made up of two components. The first is a slowly varying one caused by vibrations present in the interferometer structure, the most important one being the bending motion of the two legs (4-7 $\mathrm{Hz}$ ). The second contribution to the phase shift, of course, is chat caused by density variations in the plasma. The slow phase shift variations, i.e. those generated by pass length changes are fed into a feed back system driving a speaker carrying the reference leg retroreflecting mirror. In that way the optical path length can be kept constant, independent of mechanical vibrations in the system. The speaker can be driven such that

\footnotetext{
'On leave from FOM Institute for Atomic and Molecular Physics, Ansterdam, The Netherlands.
} 


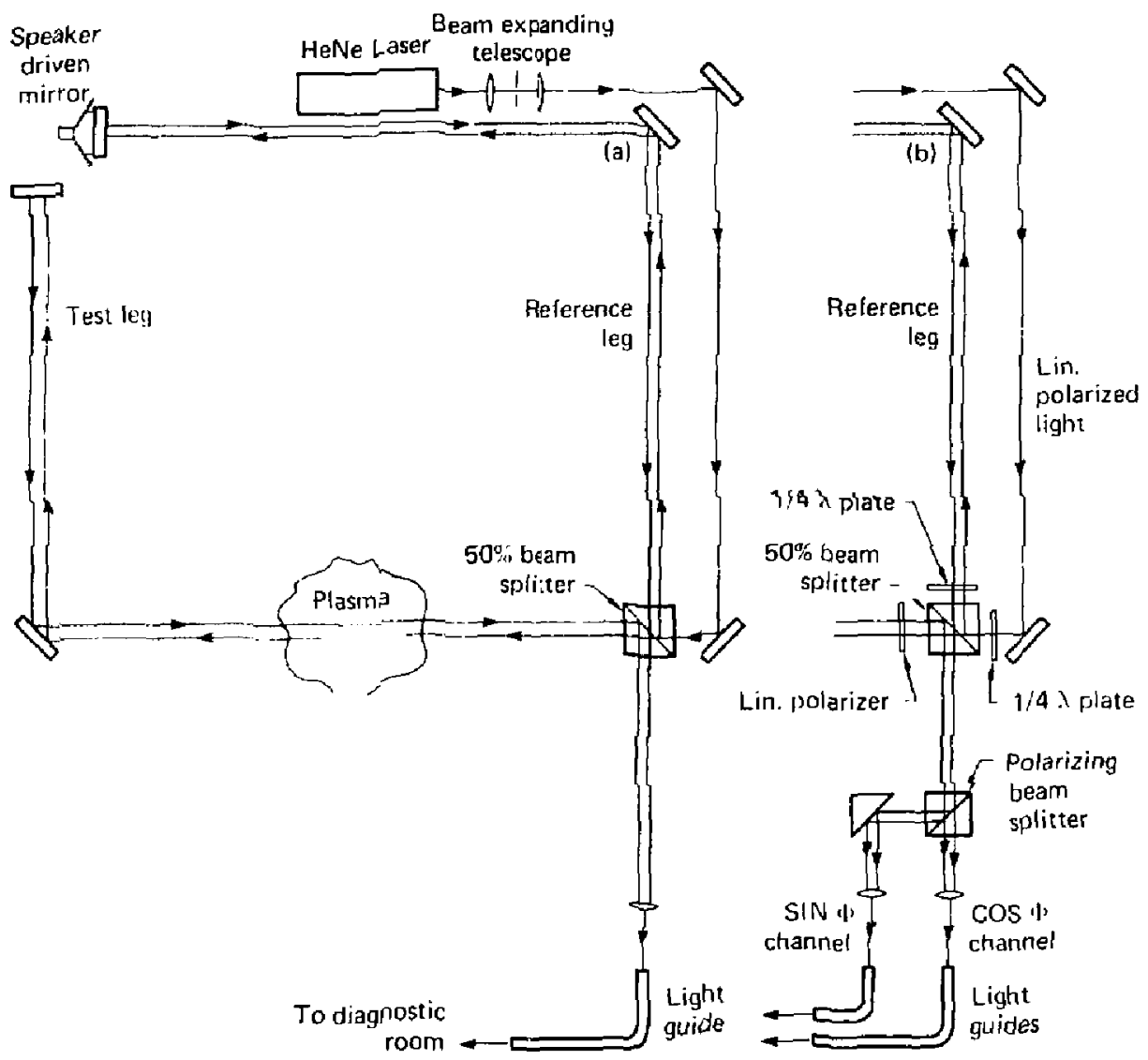

FIG. 1. The (one wavelength) interferonter as used on the BETA II experiment. (a) One channel system; (b) quadrature system $(\sin \phi, \cos \phi)$. 
for slow variations, a constant phase difference of $\pi / 2$ is maintained between reference and test beams. Hence the sensitivity (三 signal variation per unit dansity change) of the system is maximum at the moment of plasma injection. This is advantageous when the system is operated un a single channel mode (at low enougl densities, see next paragrapis).

Phase shifts of the order of $5 \times 10^{-2}$ fringe can easily be detected; depending on the experimental parameters, maxinum phase shifts corresponding with 0.2 - 1.5 fringes are commonly measured. Since ambiguities in the interpretation of the signals arise once the phase shift ( $\Phi$ ) exceeds a quarter of a fringe, two detection channels are in use registrating signals proportional to $\sin \phi$ and $\cos \phi$, respectively (for details, see 1). since in the present experiment plasma is present during approximately 400 - 800 jsec, whereas, the mechanical vibrations cause phase shifts of tie order of 0.05 fringe/ $\mathrm{msec}$, it is possible to correct for this latter contribution by measuring the "background" phase before and after the shot and interpolate during the shot. However, in the next stage of field reversed plasta ring experiments neutral beam heating of the compact torus is planned, This extends the lifetime of the plasma to possibly the 10 - 20 msec range. It is clear that the "background" phase shift caused by mechanical vibrations can no longer be determined through a simpie incerpolation procedure but has to be measured in some way. This can be done by means of an interferometer operating at two different wavelengths. Baker end Lee ${ }^{2}$ have described such a system far the Doublet III tokamak; they use two different lasers, a HeNe laser $(0.6328 \mu \mathrm{m})$ and a $\mathrm{CO}_{2}$ laser $(10.6 \mu \mathrm{m})$. The set-up described in this paper uses two wavelengths emitted by one (HeNe) laser. Also the optical lay-out is markedly different.

\section{The Two-Wavelength Interferometer:}

In case of a single pass interferometer the phase variation $\Delta \phi_{p}$ caused by a change in plasma densitiy is given by:

$$
\Delta \phi_{p}=\frac{e^{2} \lambda}{4 \pi \varepsilon_{0} m c^{2}} \int n_{e} d 1
$$

in which $e$ and $m$ are the electron charge and mass, respectively. $c$ is the speed of light, $\lambda$ the laser wavelength and $s_{0}$ the permittivity of the space. $\int n_{e} d l$ is the electron density $\left(n_{e}\right)$ integrated along the line of sight. 
If we write $N_{L}=n_{e} d l\left(m^{-2}\right)$ for the single pass line density then for a double pass interterometer:

$$
\Delta \Phi_{p}=5.62 \times 10^{-15} \lambda N_{L}=\alpha \lambda N_{L}
$$

The phase variation $\Delta Q$ caused by a relative mechanical displacement $\Delta x$ betinoding iarence and test leg can be written as:

$$
\Delta \Phi_{\mathrm{m}}=\frac{2 \pi+\Delta \mathrm{x}}{\lambda}
$$

Since for different wavelengths, $\Delta \Phi_{\mathrm{p}}$ and $\Delta \phi_{\mathrm{m}}$ are different functions of $\mathrm{N}_{L}$ and $\Delta x$ an interferometer operating at two different wavelengths simultasacusly makes it possible to determine $\Delta \Phi_{p}$ and $\Delta \Phi_{m}$ separately. If we call the total phase shift $\Delta \Phi_{t}$ we can compute $\Delta \Phi_{t 1}$, and $\Delta \phi_{t 2}$ for two different wavelengths $\lambda_{1}$, and $\lambda_{2}$, respectively.

$$
\begin{aligned}
& \Delta \phi_{t l}=\Delta \phi_{\mathrm{pl}}+\Delta \Phi_{\mathrm{ml}}=\alpha \lambda_{1} N_{\mathrm{L}}+\frac{2 \pi \cdot \Delta \mathrm{x}}{\lambda_{l}} \\
& \Delta \Phi_{\mathrm{t} 2}=\Delta \Phi_{\mathrm{p} 2}+\Delta \Phi_{\mathrm{m} 2}=\alpha \lambda_{2} \mathrm{~N}_{\mathrm{L}}+\frac{2 \pi \cdot \Delta \mathrm{x}}{\lambda_{2}}
\end{aligned}
$$

$\Delta \Phi_{\mathrm{t} l}$ and $\Delta \Phi_{\mathrm{t} 2}$ are the experimentally detertined quantities; $N_{\mathrm{L}}$ and $\Delta \mathrm{x}$ can be deduced according to:

$$
\begin{aligned}
\mathrm{lN}_{\mathrm{L}}=\frac{\Delta \Phi_{t 1} \cdot \lambda_{1}-\Delta \phi_{t 2} \cdot \lambda_{2}}{\alpha \lambda_{1}^{2}-\lambda_{2}^{2}} \\
\Delta x=\frac{\Delta \Phi_{t 1} \cdot \lambda_{2}-\Delta \Phi_{t 2} \cdot \lambda_{1}}{2 \pi \lambda_{2} / \lambda_{1}-\lambda_{1} / \lambda_{2}}
\end{aligned}
$$

A good candidate for a laser supplying two wavelengths simultaneously is the HeNe laser. It emits the following three wavelengths: $0.6328 \mu \mathrm{m}, 1.152 \mathrm{jm}$ and $3.391 \mu \mathrm{m}$. A HeNe laser with powers $210 \mathrm{~mW}$ and optics optimized for the red line $(0.6328 \mu \mathrm{m})$ will emit approximately $20 \%$ of the total power in the two other lines. One with optics optimized for one of the infrared lines usually has $30 \%$ of the power in the other IR line. ${ }^{3}$ In principle it is possible to order laser output optics suct that approximately equal powers in the desired lines are emitted. The tocal power required for convenient operation depend i strongly on the total number of optical components present in the system. A 
$15 \mathrm{mb}$ (linearly polarized) HeNe laser probably is sufficient for mast applications.

In case the combined effect of $\Delta \psi_{p}$ and $\Delta \Phi_{m}$ is such that for neither of the two wavelergths the fringe limit is reached during the shor $\left(\Delta \sigma_{t}, 2<\pi / 2\right)$ the interpretation of the signal is always unambiguous and a systerl like the one shown ir: figure $2^{2}$ suffices. However, generally this will not be the case and a quadrature system for at least one-wavelength and probably for both wavelengths is necessary. A quadrature system for one of the two wavelengths is easy to accomodate (figure $2^{b}$ ); it is essentially the same as that for a one-wavelength system (figure $1^{b}$ ). The laser light has to be linearly polarized; the $1 / 4 \lambda$ plate converts this inco circularly polariaed light. The linear polarizer in the plasma leg makes the light linearly polariced again (at a loss of a factor of two in intensicy). The polarizing beam splitter, which ideally should be placed at an angle of $45^{\circ}$ with respect th ihe polarization vector of the linearly polarized beam now yields two beams with fringe patterns $\pi / 2$ out of phase.

since the mirrors, beam splitters and windows introduce additional, spurious, phase shifts, a correction wave plate is usually necessary to tune this phase difference to exactly $\pi / 2$. For instance a $1 / 4 \lambda$ plate above the $50 \%$ bean splitter has shown to be sufficient; alternately one can place a $1 / 2 \lambda$ plate adjacent to the $1 / 4 \lambda$ plate located in the incoming beam. ${ }^{4}$ ) The arientation of the optical axis of these correction plates with respect to the light polarization direction has to be determined experimentally.

To make a quadrature system for both wdvelengths is obviously more complicated. Some of the optical components can be choosen such that the required function is independent of wavelength. E.g. for the linear polarizers and the polarizing beam splitters Glan-Thompson or similar type prisms can be used. 5 Useful optical elements to consider are Magnesium Fluoride or Lithium Niobate Rochon (or Wollaston) polarizing beam splitters since these in principle split the bean directly into the four desired components. $^{5}$ Note further that by ordering a $1 / 4 \lambda$ plate for 3.164 frm one obtains an exact $1 / 4 \lambda$ plate for $0.6328 \mu \mathrm{m}(\equiv 5 / 4 \lambda)$ and one for $3.391 \mu \mathrm{m}$ within $7 \%$.

The spurious phase shifts caused by mirror., beam splitters and other optical samponents being different for both wavelengths, one probably wants to utilize two correction wave plates at least one of which influences one wavelength exclusively. It might therefore be necessary to split the beam up 


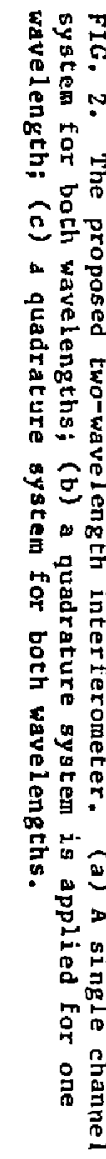

Speaker
driven Heasn expinding HeNe Laser telescope
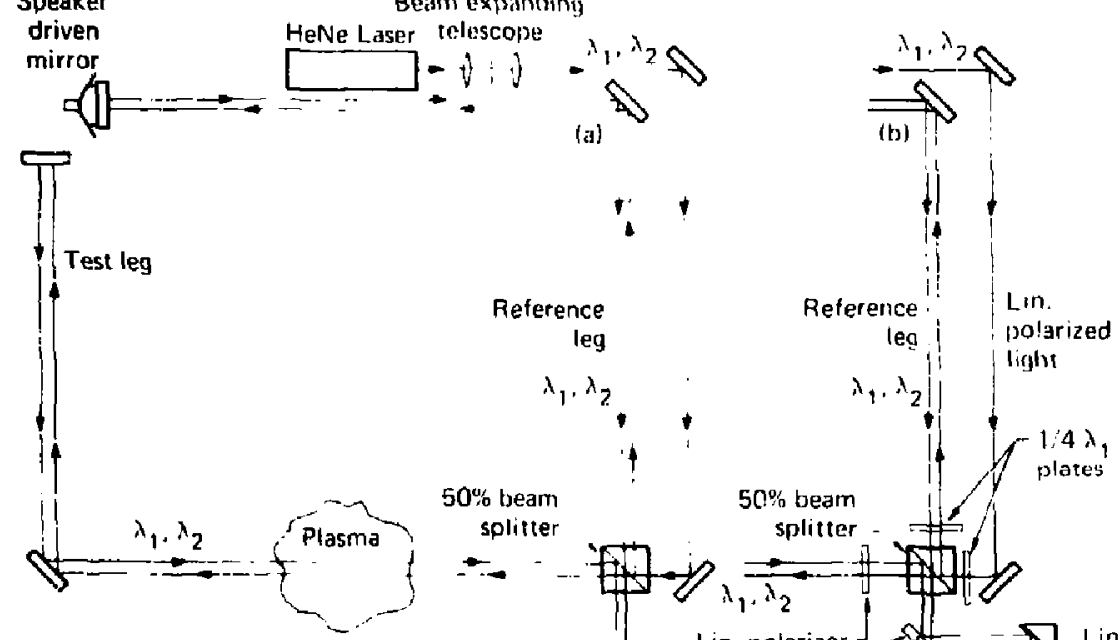

Lin. polarizer

Mirror for $\lambda_{1}$

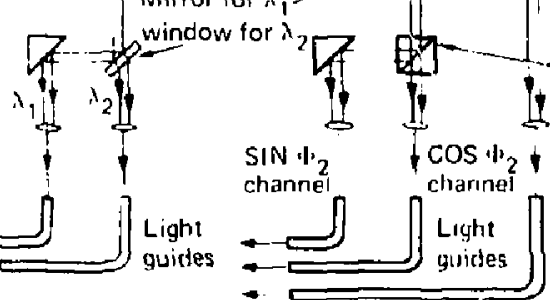

Lin. polarizer
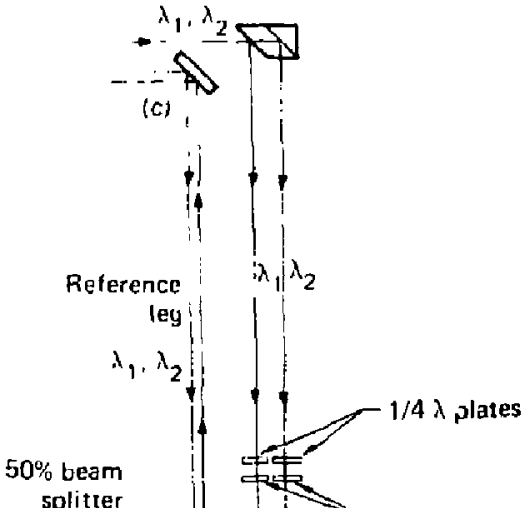
splitter 
locally (see figure $2^{c}$ ). It should be noted that the phase difference between the two fringe patterns (at one wavelength) does not have to be exactly $\pi / 2$ to remove the ambiguity in the interpretation. Any phase difference suffices in principle; however, only a $7 / 2$ phase shift yields a simple mathematical relation between the phase $\phi_{t}$ and the two recorded signals $V_{s i n}$ and $V_{c o s}: \psi_{t}=\arctan \left(V_{\sin } / V_{\cos }\right)$.

The Following numbers give an idea about the magnitude of the various phase shifts involved: At the wavelengths $0.6328,1.152$ and 3.391 bm a full fringe (2 $)$ phase shift is obtained for plasma line densities ( $\mathrm{n}_{\mathrm{e}}$ dl) equal to: $1.76 \times 10^{17}, 9.67 \times 10^{16}$ and $3.28 \times 10^{16} \mathrm{~cm}^{-2}$, respectively. Figure 3 shows phase variations caused by mechanical vibrations of the legs of the existing BETA II interferometer. Figure $3^{a}$ was recorded when the interferometer was mounted on two rubber blocks which were placed on the wooden structure surrounding the experiment. In figure $3^{\mathrm{D}}$ the interferameter was placed on an optical table which was vibrationally isolated from the wooden structure through four. NRc "air cushion" mounts."

Finally figure 4 gives examples of "experimental" signals obtained for characteristic plasma densities and mechanicai motion. The plasma line density is assumed to increase linearly during 10 us to maximun values of $10^{16}$ and $10^{17} \mathrm{~cm}^{-2}$. A 2 ter that it decays exponentially with a time constant of $10 \mathrm{~ms}$. As typical mechanical motion a sinusoidal vibration with a
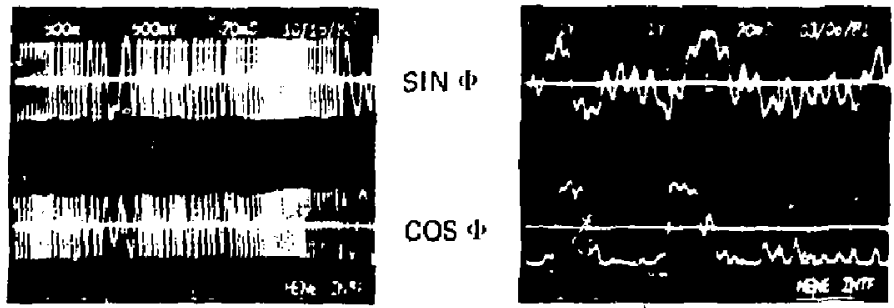

FIG. 3. The "background" fringe pattern caused by mechanical vibrations present in the interferometer structure. (a) The interferometer is mounted on two rubber blocks placed on the wooden support structure of the BETA II experiment; (b) the interferometer is mechanically isolated from the machine through four NRC vibration isolation mounts. 

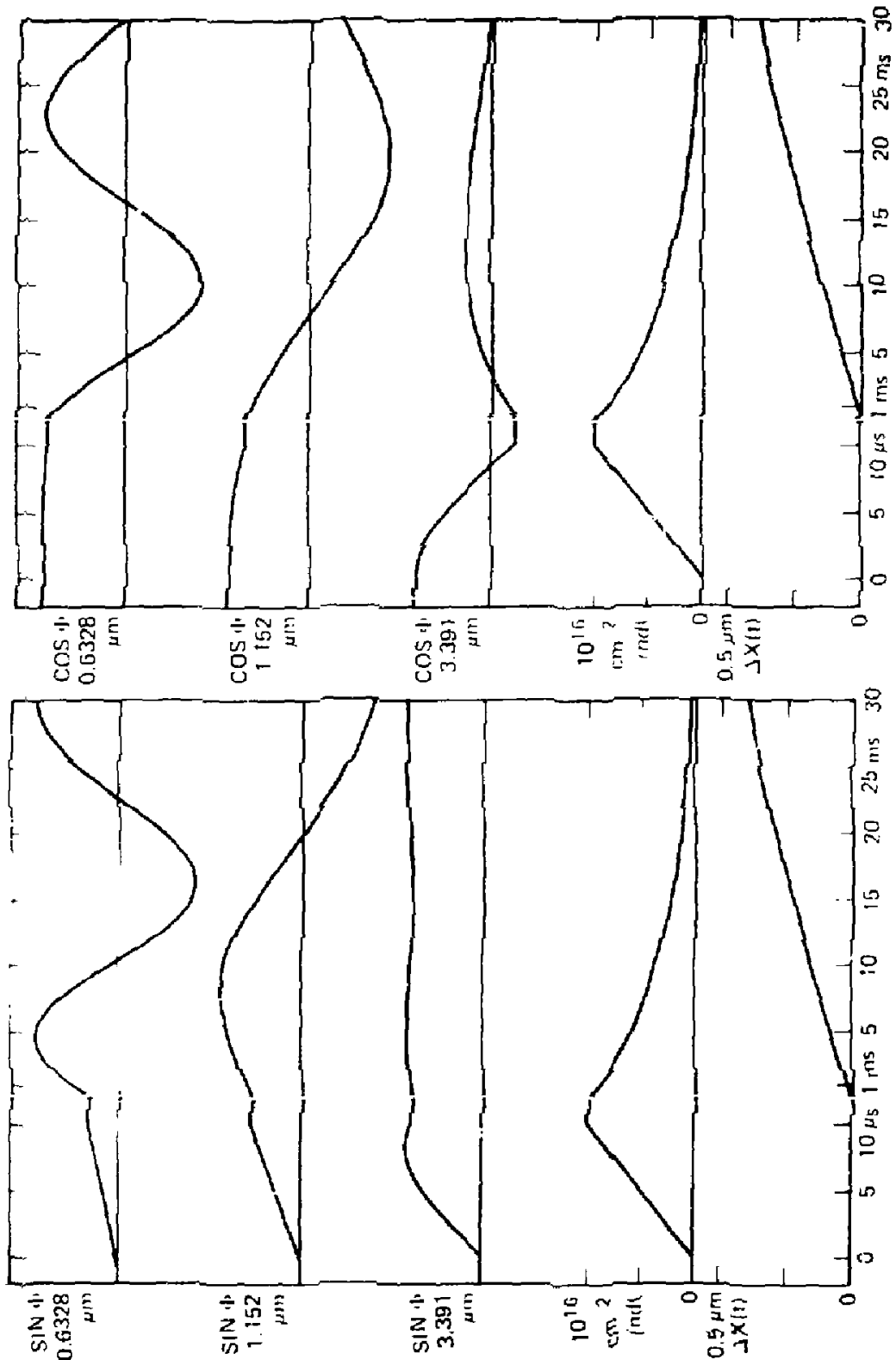

FIG. 4, Typical fringe pattern abtained for the three HeNe laser wavelengths fer a number of characteristic cases. In all cases the plasina densicy increases linearly during $10 \mu s ;$ after that it decays exponentially with a time constant of $10 \mathrm{~ms}$. The mechanical vibration is assumed to be sinusoidal with a irequency of $5 \mathrm{~Hz}$. $a, b$ : Maximum density $10^{16} \mathrm{~cm}^{-3} ; \mathrm{c}$, d: maximum density $1017 \mathrm{~cm}^{-3}$; a,c: amplicuce mechanical vibration 1 m (peak-peak); $b, d$ : arplitude mechanical vibration 5 ju (peak-peak). 


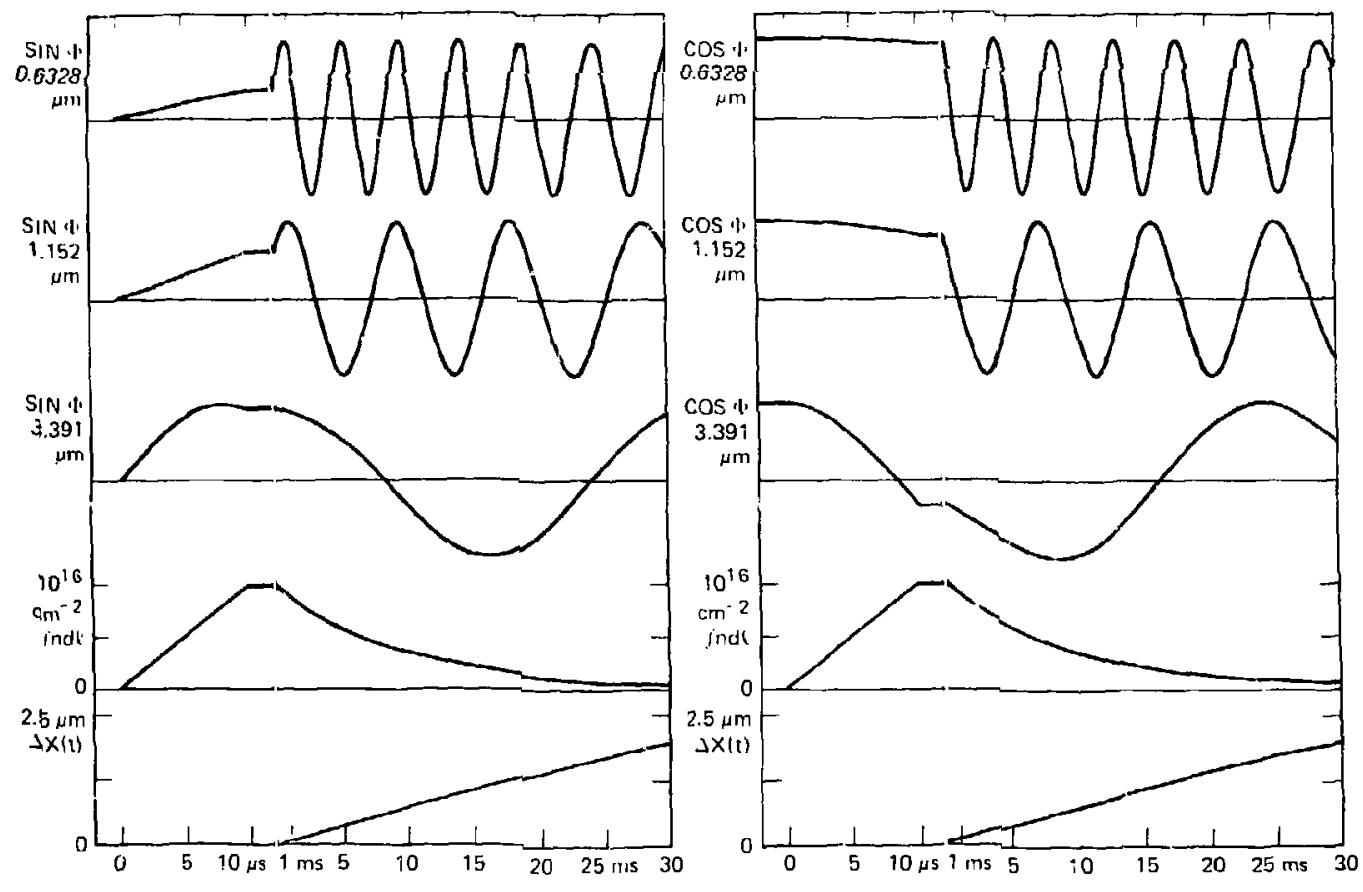



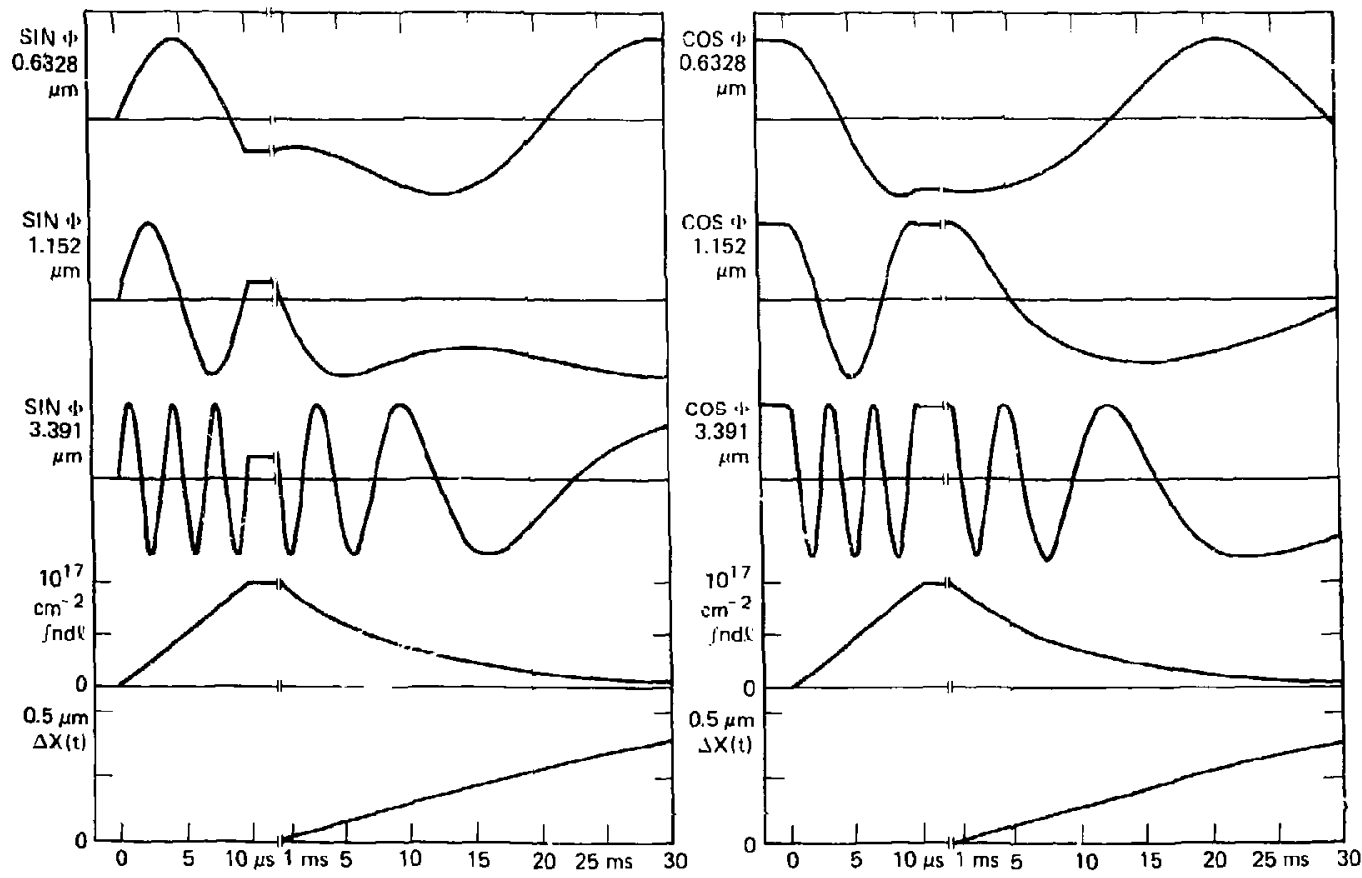

(c) 


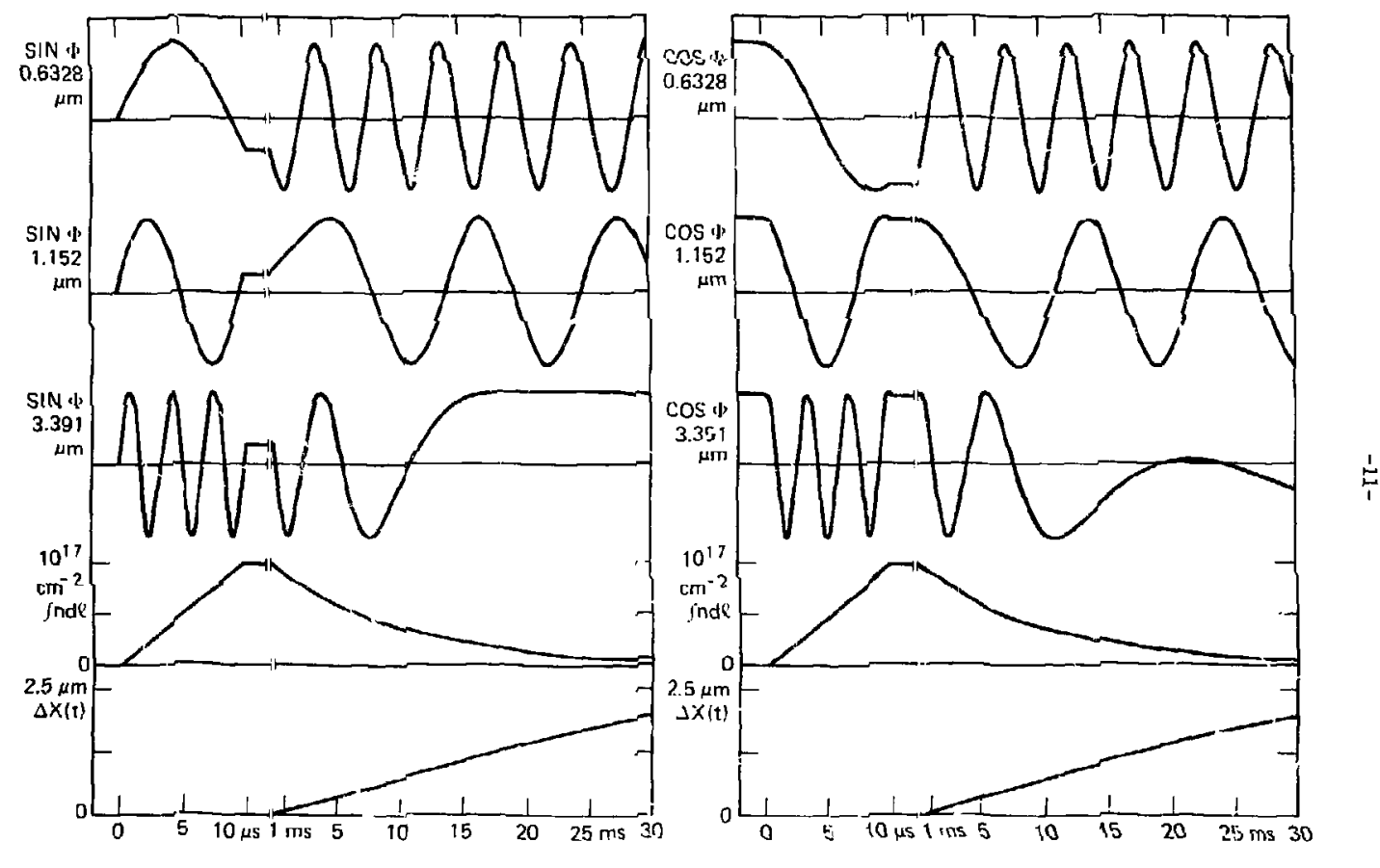


Erequency of $5 \mathrm{~Hz}$ is assumed. It starts at $t=0$ and has maximum excursions of $I$ and $5 \mu \mathrm{m}$, From these Eigures it is clear that the phase shifts induced by the plasma and by mechanical motion can be deduced from the total phase shifts measured at two different wavelengths.

\section{References}

1. Lawrence Livermore Nationil Laboratory Mirror Fusion Quarterly report, July-Saprember 1980, UCAR-10060-80-3, to be published.

2. D. R. Baker and S. I. Lee, Rev. Sc2. Instr. 49, 919 (1978).

3. Spectra Physics, Mountain View, California, private communication.

4. C. J. Buchenauer and A. R. Jacobson, Rev. Sci, Instr. 48, 769 (1977).

5. Karl Lambrecht Co., Bulletin No. P-78 (Chicago, I11., 1978).

6. Newport Research Lo. 1980-81 Catalog (Pountaia Valley, California). 